\title{
Applicability Extent of 2-D Heat Equation for Numerical Analysis of a Multiphysics Problem
}

\author{
H. Khawaja ${ }^{1, a)}$ \\ ${ }^{1}$ Associate Professor, Department of Engineering and Safety, UiT The Arctic University of Norway, Norway \\ a) Corresponding author: hassan.a.khawaja@uit.no
}

\begin{abstract}
This work focuses on thermal problems, solvable using the heat equation. The fundamental question being answered here is: what are the limits of the dimensions that will allow a 3-D thermal problem to be accurately modelled using a 2-D Heat Equation? The presented work solves 2-D and 3-D heat equations using the Finite Difference Method, also known as the Forward-Time Central-Space (FTCS) method, in MATLAB®. For this study, a cuboidal shape domain with a square cross-section is assumed. The boundary conditions are set such that there is a constant temperature at its center and outside its boundaries. The 2-D and 3-D heat equations are solved in a time dimension to develop a steady state temperature profile. The method is tested for its stability using the Courant-Friedrichs-Lewy (CFL) criteria. The results are compared by varying the thickness of the 3-D domain. The maximum error is calculated, and recommendations are given on the applicability of the 2-D heat equation.
\end{abstract}

\section{INTRODUCTION}

Engineering problems need to be discretized in space dimensions in order to be solved using numerical techniques such as finite element method (FEM), finite difference method (FDM), finite volume method (FVM), etc. [1-10]. In order to solve such numerical problems, they need to be discretized in nodes and elements [11-13]. The nodes represent the position in space where parameters are being calculated (for example, displacement, pressure, density, temperature, etc.), and elements define the equations relating to the parameters (for example, Hooke's law, laws of conservation of mass, momentum, energy, etc.) [14]. The geometry of the element defines the extent of calculations regarding space dimensions. These space dimensions categorize one-dimensional (1-D), two-dimensional (2-D) or three-dimensional (3-D) elements as shown in Fig. 1.

With respect to Fig. 1, 1-D elements are referred to as link elements and can be categorized as 2-noded or 3-noded. Similarly, 2-D elements are referred to as shells. The shape of the shell can be triangular or quadrilateral with three and four nodes respectively. If there are mid nodes within the elements, then the triangular elements can have six nodes and quadrilateral elements can consist of eight nodes. The 3-D elements can be in the shape of a tetrahedron, hexahedron, or pentahedron. Tetrahedrons have four corners, hence the same number of corner nodes, but if there are mid nodes, the total number of nodes can be up to 10 . The hexahedrons have eight nodes and, with mid nodes, there could be 20 nodes. Similarly, a pentahedron has six nodes and, with mid nodes, it has a total of 15 nodes. The shapes and positions of the elements and the number of nodes are not only limited to the aforementioned [15].

Engineering structures are referred to as 1-D, 2-D, and 3-D. An example is a piece of string, which is referred to as 1-D; in reality, however, has a cross-sectional area, which, even though is very small, has some finite value. The same concept extends to a normal size piece of paper, which is a 2-D structure; however, it has a finite value of thickness, even though it is quite small in comparison to its length and width. In fact, every structure that we can perceive is 3-D.

From the point of view of physics, it is often observed that certain physical parameters are not of significant value in a particular dimension. Let us consider a physical parameter: strength. A piece of a string is relatively strong when pulled; however, it will not provide any support in any other directions. This example can be extrapolated to a piece 
of paper: it is relatively strongly in its length and width, but it does not require much effort to bend. Therefore, from this observation, an equivalent mathematical model can be developed that limits the strength of string in 1-D and for a piece of paper in 2-D.

This physical behavior of various real time structures and physical phenomenon, allows engineers and mathematicians to assume a realistic 3-D situations to be a 2-D or even a 1-D problems. The advantage of reducing the dimensions allows solving complex equations much easier and relatively faster [1].

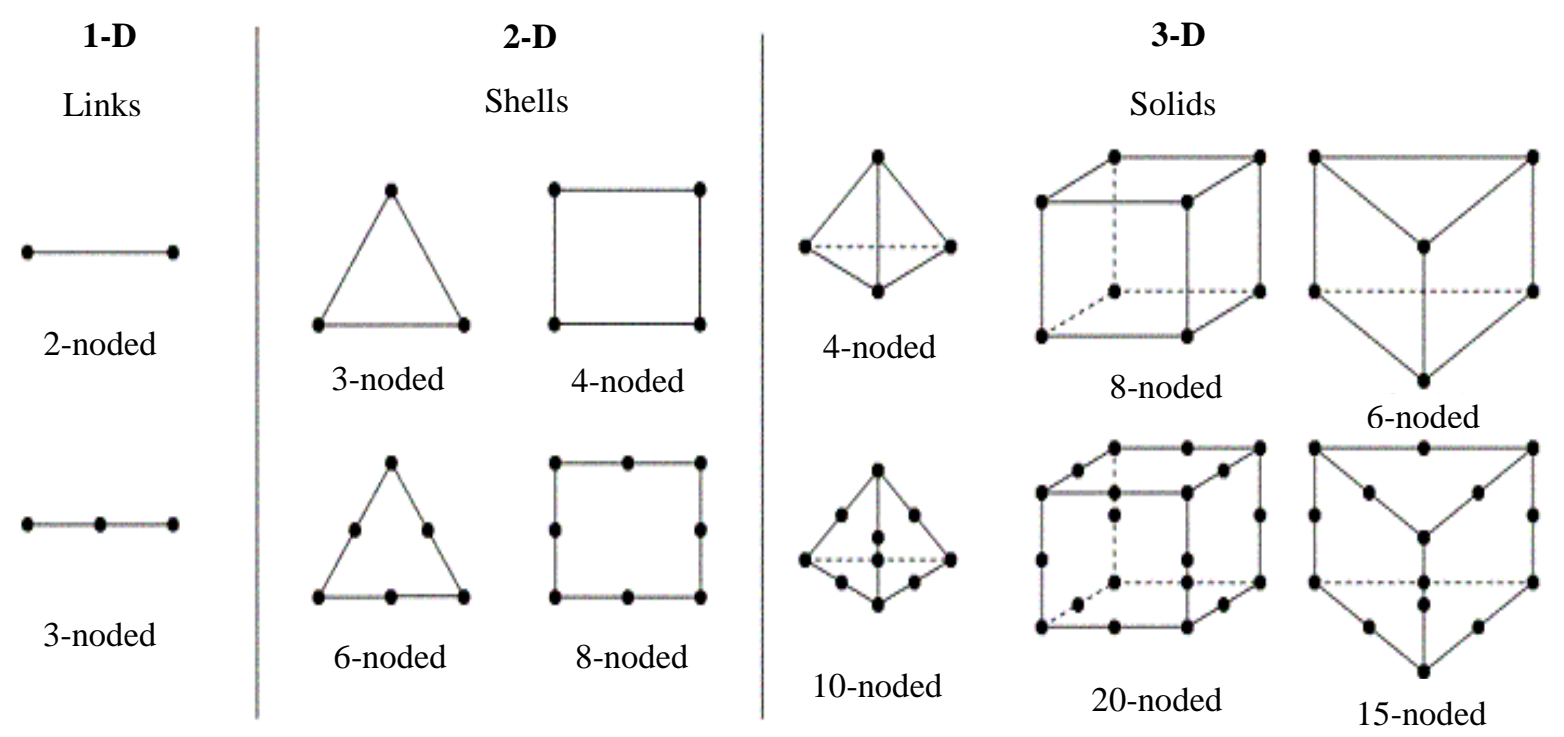

FIGURE 1. One-dimensional (1-D), two-dimensional (2-D) or three-dimensional (3-D) elements.

The given study takes a thermal problem (because of its non-linear nature) and solves it in 2-D and 3-D. This paper focuses on finding the extent of the applicability of a 2-D heat equation. An attempt is made to evaluate the error in the temperature calculations when a 3-D problem is solved via a 2-D heat equation.

\section{METHODOLOGY}

The underlying physics of heat transfer through conduction in a solid medium can be solved mathematically using the heat equation [16] as given in Equation (1):

$$
\rho c \frac{\partial T}{\partial t}=\dot{q}+\nabla \cdot(k \nabla T)
$$

where $\rho$ is the density of the medium $\left(\mathrm{kg} / \mathrm{m}^{3}\right), c$ is the specific heat capacity $(\mathrm{J} / \mathrm{kg} \mathrm{K}), \dot{q}$ is the volumetric energy generation term $\left(\mathrm{W} / \mathrm{m}^{3}\right), k$ is the coefficient of thermal conduction $(\mathrm{W} /(\mathrm{m} . \mathrm{K})), T$ is the temperature $(\mathrm{K}), \nabla$ is the del operator and $t$ is time (s).

The other forms of the above equation in two and three spatial dimensions with no energy generation term [17] are given in Equation (2) and Equation (3):

$$
\frac{\partial T}{\partial t}=\alpha\left(\frac{\partial^{2} T}{\partial x^{2}}+\frac{\partial^{2} T}{\partial y^{2}}\right)
$$




$$
\frac{\partial T}{\partial t}=\alpha\left(\frac{\partial^{2} T}{\partial x^{2}}+\frac{\partial^{2} T}{\partial y^{2}}+\frac{\partial^{2} T}{\partial z^{2}}\right)
$$

where $x, y$ and $z$ refer to spatial positions $(\mathrm{m})$ in three dimensions and $\alpha$ is the thermal diffusivity term $\left(\mathrm{m}^{2} / \mathrm{s}\right)$ as given in Equation (4):

$$
\alpha=\frac{k}{\rho c}
$$

A finite difference method (FDM) is a numerical method for solving differential equations such as the heat equation given in Equation (2) and Equation (3). This method approximates the differentials with differences by discretizing the dependent variable (temperature) in the independent variable domains (space and time) [18]. Each discretized value of the dependent variable is referred to as a nodal value. In this case, the heat equations given in Equation (2) and Equation (3) are discretized using the FDM, forward-time central-space (FTCS). The discretized equations in two and three dimensions are given in Equation (5) and Equation (6).

$$
\begin{aligned}
T_{i, j, k}^{t+1}= & T_{i, j, k}^{t}+\alpha \frac{\left(T_{i+1, j, k}^{t}-2 T_{i, j, k}^{t}+T_{i-1, j, k}^{t}\right)}{(\Delta x)^{2}} \Delta t \\
& +\alpha \frac{\left(T_{i, j+1, k}^{t}-2 T_{i, j, k}^{t}+T_{i, j-1, k}^{t}\right)}{(\Delta y)^{2}} \Delta t \\
T_{i, j, k}^{t+1}= & T_{i, j, k}^{t}+\alpha \frac{\left(T_{i+1, j, k}^{t}-2 T_{i, j, k}^{t}+T_{i-1, j, k}^{t}\right)}{(\Delta x)^{2}} \Delta t \\
& +\alpha \frac{\left(T_{i, j+1, k}^{t}-2 T_{i, j, k}^{t}+T_{i, j-1, k}^{t}\right)}{(\Delta y)^{2}} \Delta t \\
& +\alpha \frac{\left(T_{i, j, k+1}^{t}-2 T_{i, j, k}^{t}+T_{i, j, k-1}^{t}\right)}{(\Delta z)^{2}} \Delta t
\end{aligned}
$$

where superscript $t$ and subscript $i, j, k$ refer to time and position respectively. $\Delta t$ is the time step size (s), and $\Delta x, \Delta y, \Delta z$ are the differences in the spatial positions of temperature nodes.

To solve Equations (5) or (6), initial and boundary conditions are required. Constant temperature boundary conditions were applied to the outer boundaries in both of the cases. A constant temperature of $273 \mathrm{~K}$ was specified for the $x$ and $y$ edges as boundary conditions in the case of the 2-D problem, as given in Equation (7) and illustrated in Figure 2. In the case of 3-D, a constant temperature of $273 \mathrm{~K}$ was specified for the $x$ and $y$ surfaces, and zero thermal derivative was specified for the $z$ surfaces, as given in Equation (8) and illustrated in Figure 2. The zero thermal derivative ensures that there are no heat losses in the z-direction, which is consistent with the 2-D problem.

$$
\begin{gathered}
T_{1, j}=273 ; T_{i, 1}=273 ; T_{\max -x, j}=273 ; T_{i, \max -y}=273 ; \\
T_{1, j, k}=273 ; T_{i, 1, k}=273 ; \frac{\partial T_{i, j, 1}}{\partial z}=0 ; T_{\max -x, j, k}=273 ; T_{i, \max -y, k}=273 ; \frac{\partial T_{i, j, \max -z}}{\partial z}=0 ;
\end{gathered}
$$

where $\max -x, \max -y$ and $\max -z$ are the domain space limits in $x, y$ and $z$ dimensions, respectively. 
A constant temperature heat source of $298 \mathrm{~K}$ was specified in the middle of the 2-D and 3-D domains as illustrated in Figure 2.

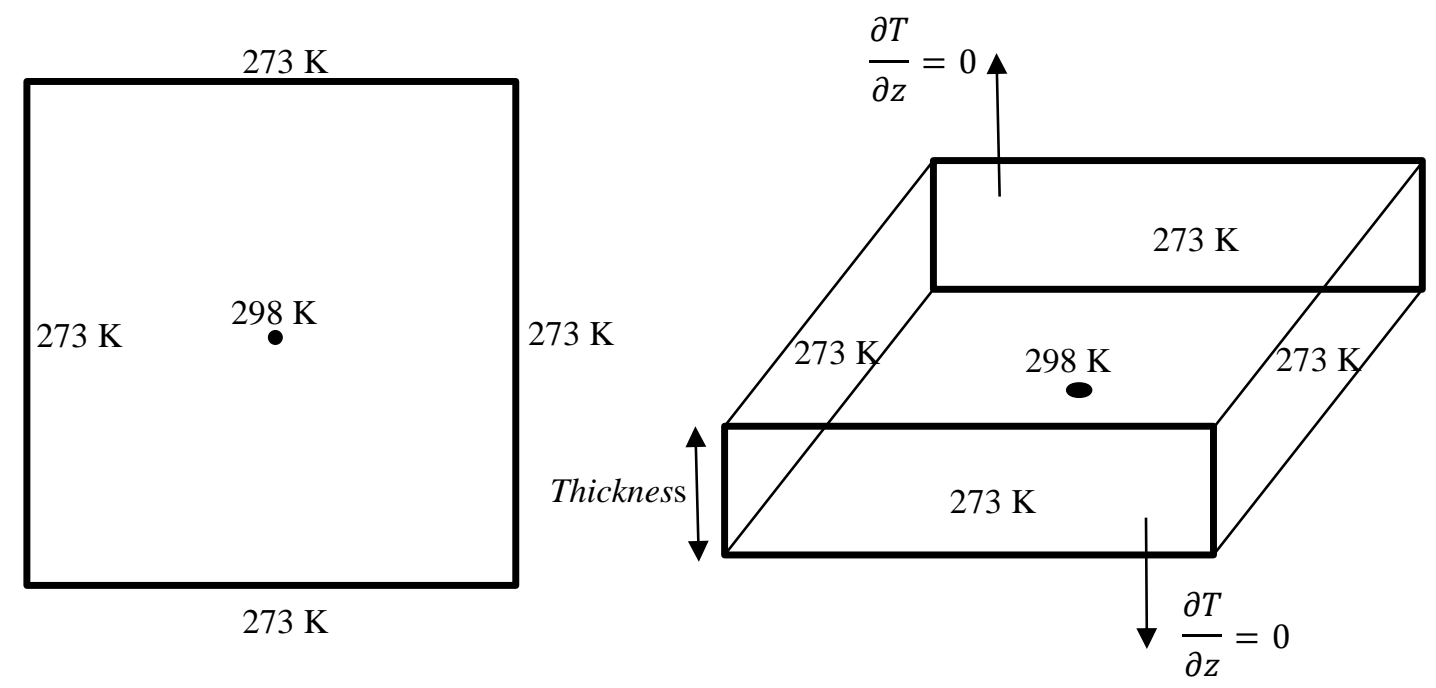

FIGURE 2. Boundary conditions applied in the 2-D and 3-D cases.

The domains (2-D and 3-D) are discretized, as shown in Figure 3.
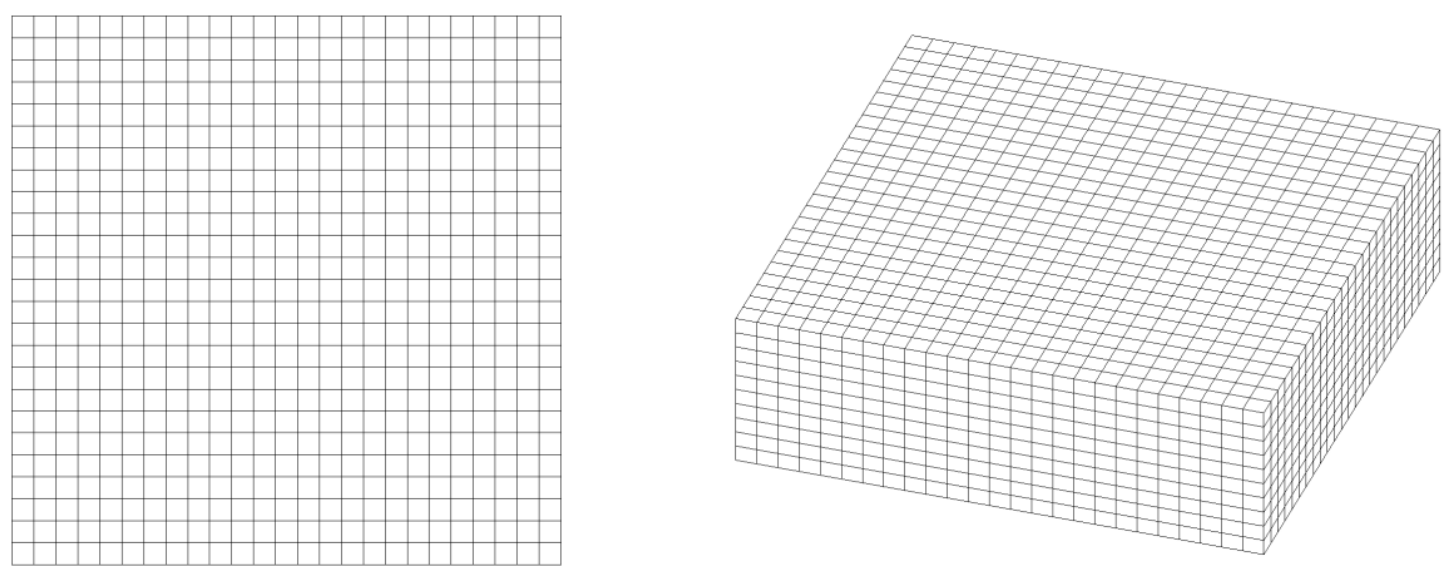

FIGURE 3. The discretized 2-D and 3-D domains.

As an initial condition, a constant temperature of $273 \mathrm{~K}$ was specified throughout the domain, except at the source. The initial condition was the same for 2-D and 3-D cases. The size of the 2-D space is $250 \mathrm{~mm} \mathrm{X} 250 \mathrm{~mm}$, and the size of the 3-D space is $250 \mathrm{~mm}$ X $250 \mathrm{~mm}$ X thickness.

The study focuses on the variable Thickness in the 3-D case. The material properties are given in Table 1. 
TABLE 1. Values of constants and coefficients in heat equation.

\begin{tabular}{ccc}
\hline Constant & Value & Units \\
\hline Density $(\rho)$ & 100 & $\mathrm{~kg} / \mathrm{m}^{3}$ \\
Specific Heat Capacity $(c)$ & 2000 & $\mathrm{~J} /(\mathrm{kg} . \mathrm{K})$ \\
Coefficient of Thermal Conductivity $(k)$ & 1 & $\mathrm{~W} /(\mathrm{m} . \mathrm{K})$ \\
Thermal Diffusivity $(\alpha)$ & $5.0 \times 10^{-6}$ & $\mathrm{~m}^{2} / \mathrm{s}$ \\
\hline
\end{tabular}

It is vital for the stability and accuracy of the FDM to choose the correct time step value. In this work, the CourantFriedrichs-Lewy (CFL) condition $[18,19]$ is used to decide the time step size. The CFL condition for the heat equation is given in Equation (9):

$$
2 \alpha \Delta t \leq \min \left((\Delta x)^{2},(\Delta y)^{2},(\Delta z)^{2}\right)
$$

where $\alpha$ is the thermal diffusivity term $\left(\mathrm{m}^{2} / \mathrm{s}\right), \Delta t$ is the time step size (s) and $\Delta x, \Delta y, \Delta z$ are the differences in the spatial positions of the temperature nodes.

Equations (5) and (6) are solved and post-processed in MATLAB ${ }^{\circledR}[20]$. The flow chart of the 2-D and 3-D codes is given in Figure 4. The results are discussed in the next section.

\section{RESULTS AND DISCUSSION}

The steady-state temperature profile of the 2-D case is given in Figure 5. The figure shows that the temperature is $298 \mathrm{~K}$ in the center, gradually reducing to $273 \mathrm{~K}$ at the boundaries. Close to the center, the isothermal lines are circular and become square shaped close to the boundaries. The temperature variation from edge to edge of the 2-D space (passing through the center) is given in Figure 6. The maximum temperature is $298 \mathrm{~K}$, which is in the center. The temperature drops parabolically to $273 \mathrm{~K}$ on either side, as expected from the solution of the heat equation [21, 22].

The parabolic behavior was also observed in the 3-D space. However, the parabolic behavior differs with different thicknesses, as shown in Figure 7.

To quantify the error, the maximum temperature difference was calculated within 2-D and 3-D cases. The error values were normalized against maximum possible temperature difference $(298-273=25 \mathrm{~K})$. Similarly, the thickness was also normalized against the $\mathrm{x}$ dimension. It is to be noted that, for the simplicity of this analysis, the $\mathrm{x}$ and $\mathrm{y}$ dimensions are the same.

The percentage errors with normalized thicknesses are given in Figure 8. 


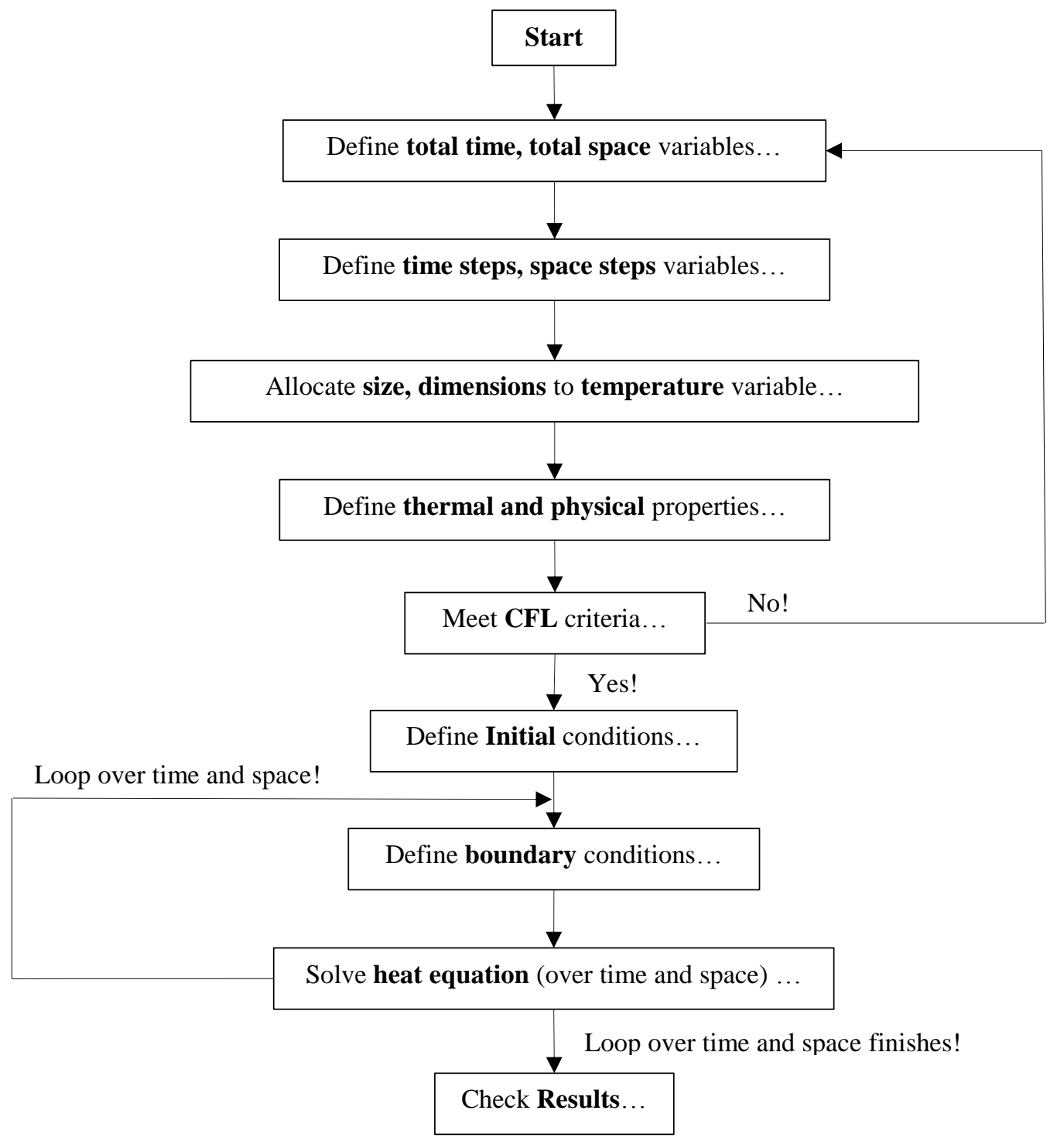

FIGURE 4. Boundary conditions for the 2-D and 3-D cases. 


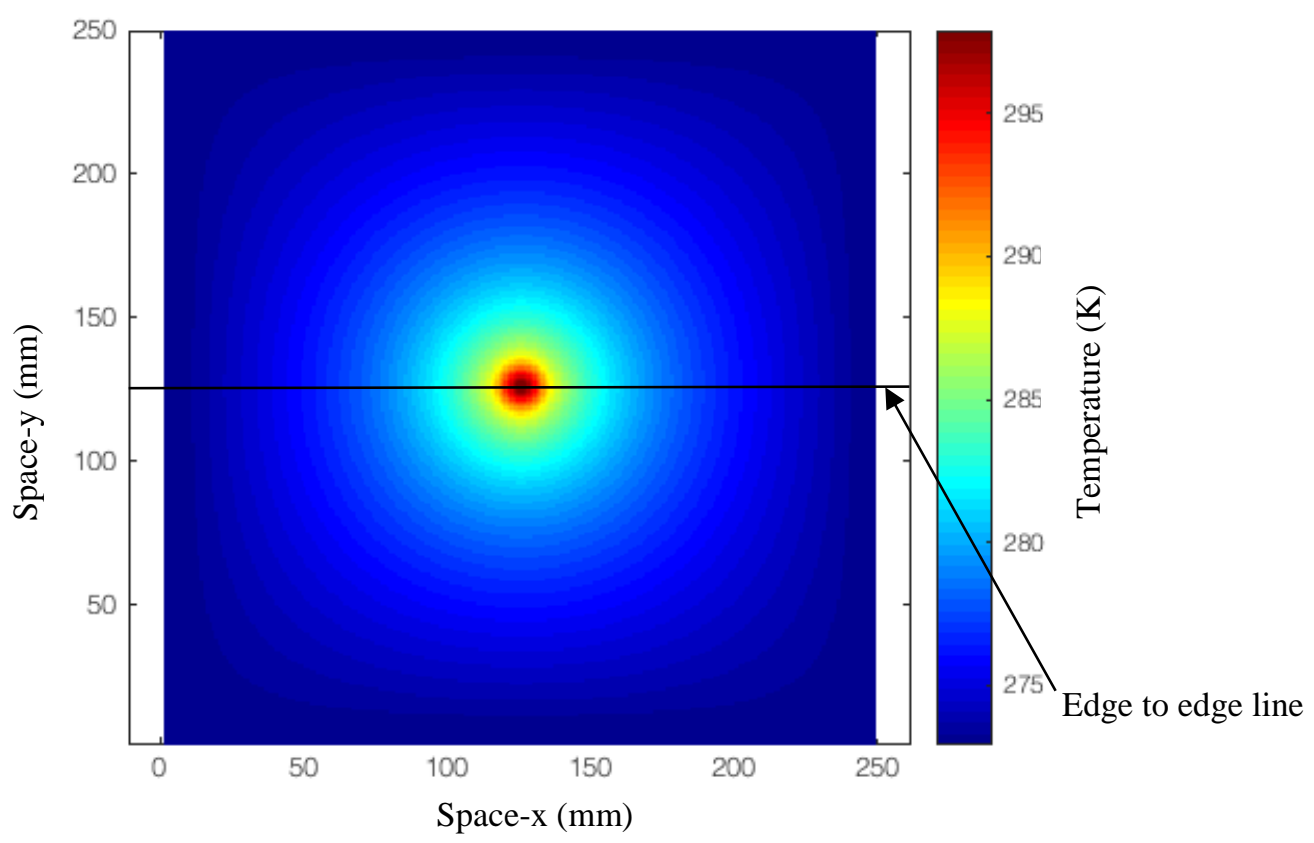

FIGURE 5. The temperature profile of 2-D space.

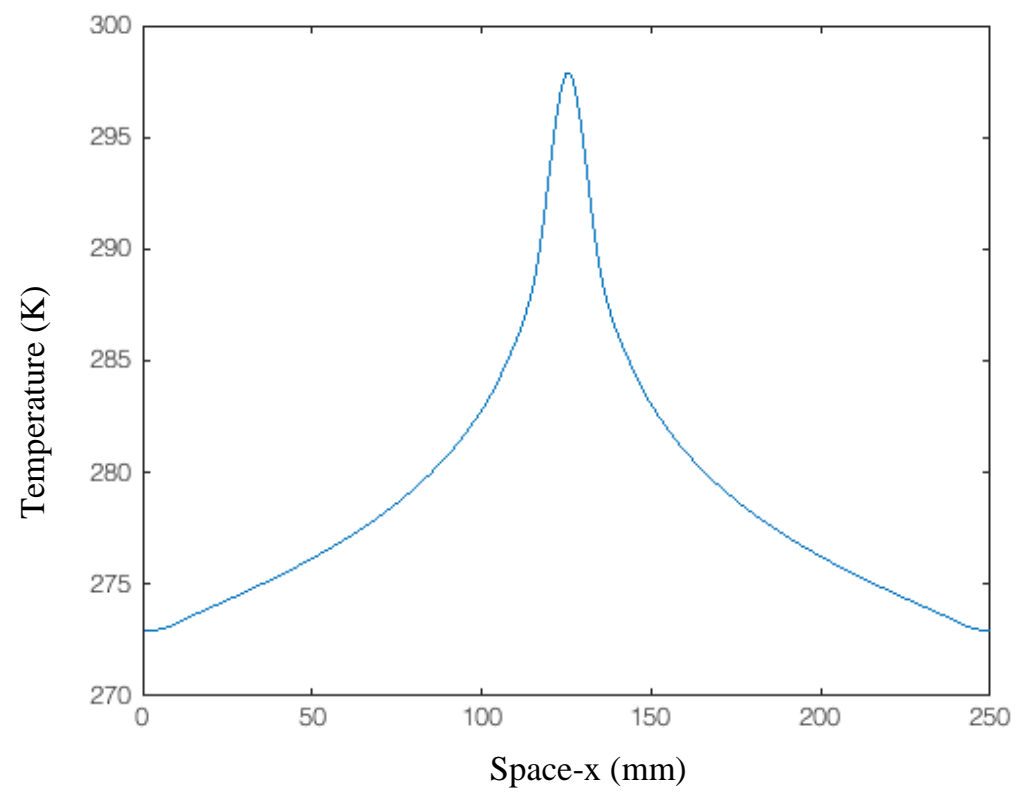

FIGURE 6. Temperature variation in 2-D space on the edge to edge line passing through the center. 


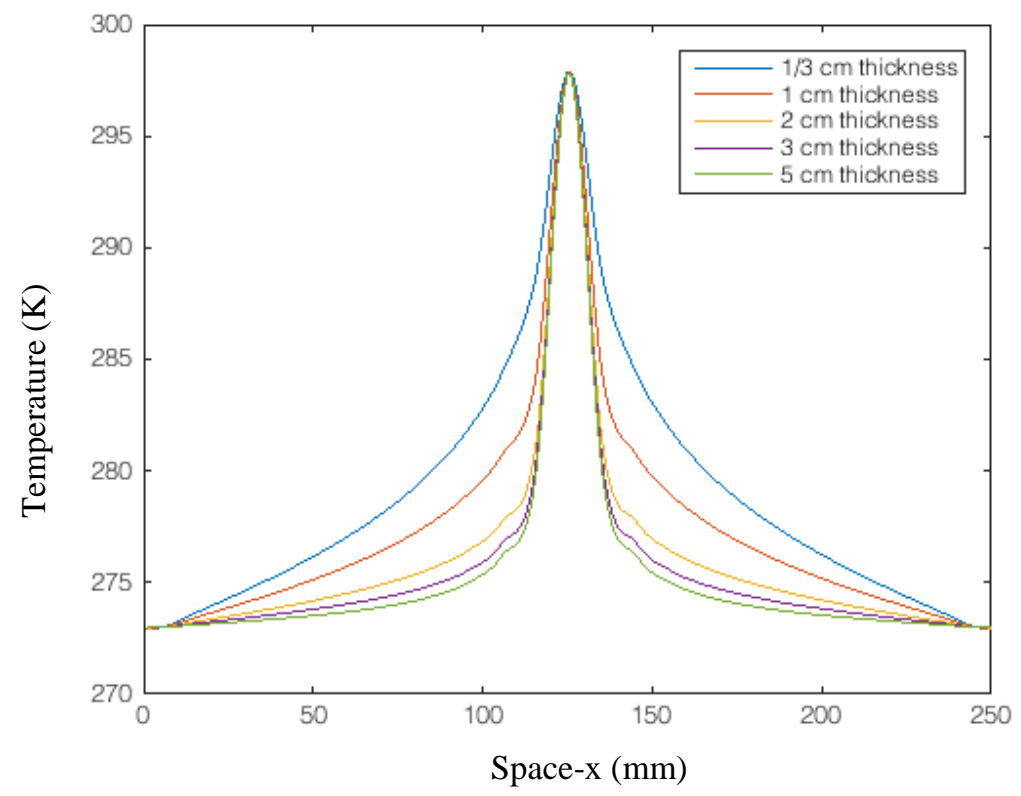

FIGURE 7. Temperature variation in 3-D space on the edge to edge line passing through the center.

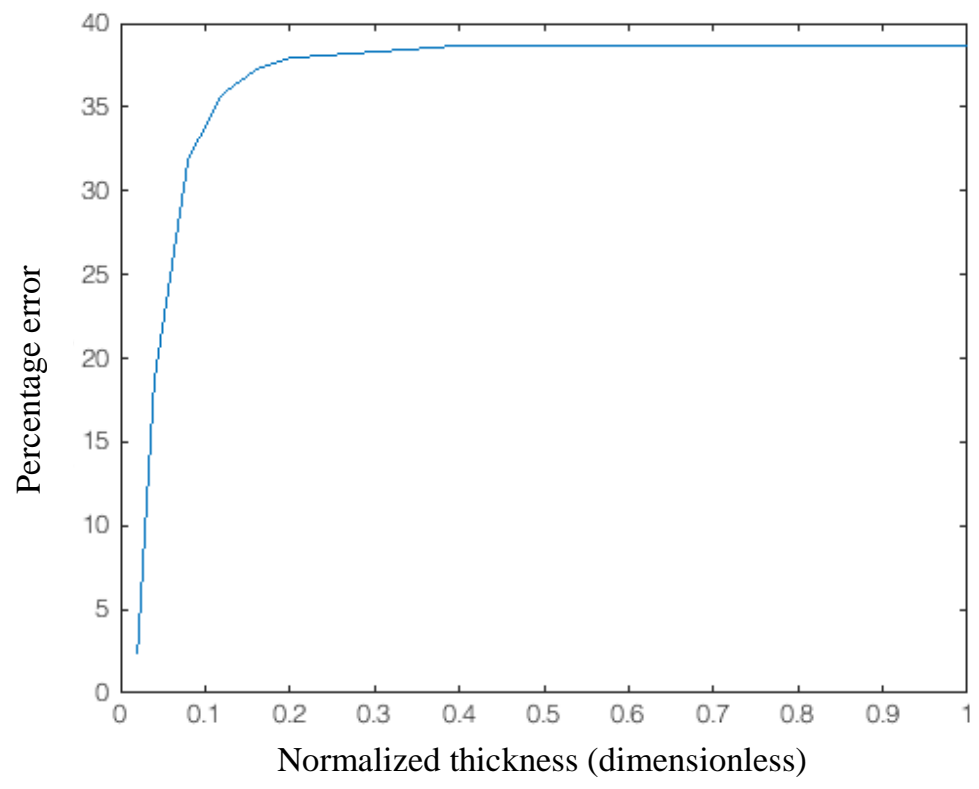

FIGURE 8. Percentage error between 2-D and 3-D heat transfer cases.

\section{CONCLUSION}

The presented study demonstrates the extent of error when a 2-D heat equation is used to solve a 3-D problem. The study includes a partial differential (PDE) heat equation, which is solved using the forward-time centered-space (FTCS) finite difference method (FDM). It is concluded that the error is small in the solution of the 2-D heat equation 
when the thickness is small in comparison to the other dimensions (normalized thickness to be around 0.05). The error values rise sharply for higher normalized thickness values. This study showed the maximum error to be about $38 \%$; however, this value can differ, depending on the problem domain, physical properties, initial and boundary conditions.

\section{ACKNOWLEDGMENTS}

The author is grateful for the input from MS students, who took part in the TEK-3015 Multiphysics Simulation course in spring 2016. The author would also like to acknowledge the support of Linda March from The Good English Company, UK for proofreading this work.

\section{REFERENCES}

[1] H. Khawaja. Application of a 2-D approximation technique for solving stress analyses problem in FEM. The International Journal of Multiphysics. 2015;9(4):317-24.

[2] H. Khawaja. CFD-DEM Simulation of Minimum Fluidisation Velocity in Two Phase Medium. The International Journal of Multiphysics. 2011;5(2):89-100.

[3] H. Khawaja, S. Scott. CFD-DEM Simulation of Propagation of Sound Waves in Fluid Particles Fluidised Medium. The International Journal of Multiphysics. 2011;5(1):47-60.

[4] H. Khawaja, S. Scott, M. Virk, M. Moatamedi. Quantitative Analysis of Accuracy of Voidage Computations in CFD-DEM Simulations. The Journal of Computational Multiphase Flows. 2012;4(2):183-92.

[5] H. Khawaja, I. Raouf, K. Parvez, A. Scherer. Optimization of elstomeric micro-fluidic valve dimensions using non-linear finite element methods. The International Journal of Multiphysics. 2009;3(2):187-200.

[6] H. Khawaja, K. Parvez. Validation of normal and frictional contact models of spherical bodies by FEM analysis. The International Journal of Multiphysics. 2010;4(2):175-85.

[7] H. Khawaja, M. Moatamedi. Selection of High Performance Alloy for Gas Turbine Blade Using Multiphysics Analysis. The International Journal of Multiphysics. 2014;8(1):91-100.

[8] H. Khawaja, T. Bertelsen, R. Andreassen, M. Moatamedi. Study of CRFP Shell Structures under Dynamic Loading in Shock Tube Setup. Journal of Structures. 2014;2014(Article ID 487809):6.

[9] H. Khawaja, R. Messahel, B. Ewan, S. Mhamed, M. Moatamedi. Experimental and Numerical Study of Pressure in a Shock Tube. Journal of Pressure Vessel Technology. 2016;138(4):6.

[10] U. Mughal, H. Khawaja, M. Moatamedi. Finite element analysis of human femur bone. The International Journal of Multiphysics. 2015;9(2):101-8.

[11] M. Kim. Finite Element Methods with Programming and Ansys: LULU Press; 2013.

[12] T. Stolarski, Y. Nakasone, S. Yoshimoto. Engineering Analysis with ANSYS Software: Elsevier Science; 2011.

[13] Y. W. Kwon, H. Bang. The Finite Element Method Using MATLAB, Second Edition: CRC Press; 2000.

[14] H. D. Young, A. L. Ford, R. A. Freedman. University Physics: Pearson Education; 2008.

[15] Y. W. Kwon. Multiphysics and Multiscale Modeling: Techniques and Applications: CRC Press; 2016.

[16] J. R. Cannon. The One-Dimensional Heat Equation: Cambridge University Press; 1984.

[17] D. V. Widder. The Heat Equation: Elsevier Science; 1976.

[18] S. Patankar. Numerical Heat Transfer and Fluid Flow: Taylor \& Francis; 1980.

[19] R. Courant, K. Friedrichs, H. Lewy. Über die partiellen Differenzengleichungen der mathematischen Physik. Mathematische Annalen. 1928;100(1):32-74.

[20] MATLAB®. Natick, Massachusetts: The MathWorks Inc.; 2016.

[21] H. Khawaja, T. Rashid, O. Eiksund, E. Brodal, K. Edvardsen. Multiphysics Simulation of Infrared Signature of an Ice Cube. The International Journal of Multiphysics. 2016;10(3):In press.

[22] T. Rashid, H. Khawaja, K. Edvardsen. Determination of Thermal Properties of Fresh Water and Sea Water Ice using Multiphysics Analysis. The International Journal of Multiphysics. 2016;10(3):In press. 